\title{
Estrategias Financieras para el Mejoramiento del Valor Económico Agregado
}

\author{
Percy Vilchez Olivares \\ Docente de la Facultad de Ciencias Contables
}

\begin{abstract}
RESUMEN
El Valor Económico Agregado es la medida financiera que viene revolucionando en el mundo de los negocios, puesto que permite a las empresas que lo implantan, optimizar la gestión empresarial y maximizar la riqueza de los accionistas.

Esta ponencia se compone de varias partes. En la primera se presentan temas de economía y finanzas en el mundo globalizado y su vinculación con la creación de valor. Después se revisa la vinculación que tiene la gestión empresarial y las medidas financieras. Asimismo, se estudia el marco conceptual del Valor Económico Agregado, las finanzas y las estrategias empresariales, y el impacto que tienen las estrategias financieras para aumentar el Valor Económico Agregado. Después se presenta una investigación aplicada a una empresa peruana que ha desarrollado estrategias financieras para optimizar la gestión empresarial y maximizar la genetación de valor en la empresa. En la parte final se incluye conclusiones de la ponencia.

Con todo ello, se espera contribuir a introducir el concepto de Valor Económico Agregado como medida financiera y presentar un enfoque analítico y sistemático para orientar el esfuerzo de transformación económica de la empresa hacia la optimización de la gestión y maximizar la riqueza de los accionistas.
\end{abstract}

Palabras clave: Valor económico agregado, globalización de los mercados, nueva economía.

\section{INTRODUCCION}

Con la presencia de un nuevo orden económico, denominado la Nueva Economía, la cual es el resultado de tendencias (fuerzas) que generan cambios significativos en los modosyprocesos económicosyempresariales, aparecen diversas macrotendencias. Estas macrotendencias como la globalización de los mercados, el desarrollo acelerado de la tecnología, la liberalización y privatización de la economía, la importancia de los activos intangibles; hacen que el éxito de las organizaciones empresariales y la riqueza de los países dependan cada vez menos de las ventajas comparativas (recursos naturales, salarios) de las naciones, sino de la productividad de la mano de obra y la tecnología.
El desarrollo económico de los países de América Latina y el crecimiento sostenido de las organizaciones empresariales dependen crecientemente más de la creación eficiente de valor. Es esta creación (producción con valor agregado) la que permitirá ser competitivos en calidad y precios en los ámbitos nacional e internacional. La posibilidad de competir exitosamente en los mercados nacionales $\mathrm{e}$ internacionales crece conforme se incrementa la capacidad para producir bienes y servicios; y aumenta el aprendizaje organizacional que generan mayor valor, tanto para el cliente como para los demás actores de la gestión empresarial: accionistas, directores, gerentes, personal, proveedores, el Estado y la sociedad. A esto se denomina creación de Valor. 
Al respecto, Alejandro Indacochea (1998; pp. 29:31), en la Convención Empresarial de ESAN (1997), señala «la competitividad y la creación de Valor son temas que tienen una vinculación directa. Un país crea valor y es competitivo si es exitoso en el Comercio Internacional, es decir, capaz de colocar bienes o servicios mejores y más baratos que los de la competencia; aprovechando el uso adecuado del conocimiento (educación, investigación y desarrollo, y tecnología) y a una alta productividad (eficiencia más eficacia), y si con ello genera elevados niveles de vida para la población». En ese sentido, precisa que «para la creación de Valor, adicionalmente se debe cumplir algunos requisitos básicos: mano de obra calificada, favorable ambiente de negocios, tecnologia e infraestructura apropiadas; con esos elementos, el país puede tener mayor competitividad y productividad».

Por lo expuesto, debo precisar la creación de Valor y la capacidad competitiva de las economías nacionales, es un aspecto que esencialmente compete a las empresas que son los agentes económicos centrales de la creación de Valor y del Comercio Internacional y son las que, finalmente, están obligadas a enfrentar la competencia. Sin embargo, debo puntualizar que el enfoque debe ir más allá de la responsabilidad económica y social de las empresas, pues es un asunto de interés nacional.

Por lo expuesto, debo precisar que la competitividad y la creación de Valor para el país es un compromiso que necesita del esfuerzo y la concertación de todos los agentes económicos, para iniciar el desarrollo de transformaciones sostenibles y profundas en las economías de las naciones de América Latina.

\section{LAS FINANZAS EN UN MUNDO GLOBALIZADO}

El fenómeno de la globalización de la economía y sus manifestaciones expuestas principalmente, por la continua globalización de los negocios y la globalización de inversiones; así como la tecnología relacionada con el conocimiento; hacen repensar que en el nuevo orden mundial nada puede ser hecho sin tener en cuenta el contexto global.

La globalización implica la difusión de los patrones de referencia mundiales, al margen de las distancias geográficas, tiene varios conceptos sobre el mismo. Al respecto, el Informe del Grupo de Lisboa integrado por pensadores de Europa, Estados Unidos y Japón (1995, p. 20), señala que los conceptos de globalización son la globalización de las finanzas, mercado y estrategias, tecnología vinculada al conocimiento, cultura, gobierno y las percepciones.

Un aspecto central de la globalización financiera es que ha originado la pérdida de la procedencia de la inversión que se realiza en un país; es decir, cuando el capital es nacional o extranjero. De acuerdo a un informe de la Cámara de Comercio de Santiago; la inversión chilena en el Perú para el período 1990 al 2004 es de cuatro mil treinta y ocho millones de dólares. Pero gran parte de esta inversión chilena no vino directamente de Chile, sino a través de los centros «offshore», o paraísos fiscales: Aruba, Bahamas, Islas Caimán; en donde mediante una cuenta cifrada muchas veces es imposible saber quién es el verdadero propietario del capital. Esta es una peculiaridad del fenómeno de globalización financiera. 
Otro aspecto importante es que la globalización financiera es la dimensión de las economias y empresas. En la actualidad, Estados Unidos, Europa, Japón y los países industrializados del sur este Asiático, concentran más del $70 \%$ de la actividad económica mundial; esto es importante para dimensionar la economía de cada país de América Latina. Lo anterior influye en el desarrollo de las corporaciones en los países, industrializados y desarrollados en que interactúan. Al respecto, la Revista Business (2004, pp. 30:31) publicó una lista de las corporaciones mundiales más grandes, según la revista Forbes y Fortune al 2003, la cual permite identificar corporaciones con niveles de ingresos y valor de mercado superiores al PBI de muchos países de América Latina; y que a continuación se detallan algunas:

Las mayores corporaciones americanas tienen ventas totales Euporiores al PBI de muchos paises de América Latina

\begin{tabular}{|c|c|c|c|}
\hline \multirow[b]{2}{*}{ Compañiี } & \multicolumn{3}{|c|}{ Miles de millones US \$ } \\
\hline & Industria & Ventas & Valor Mercado \\
\hline General Electric & Conglomerados & 134 & 329 \\
\hline Exxon Mobil & Petróleo ygas & $\rightarrow 213$ & 281 \\
\hline Wal Mar Stores & Minorista & 259 & 264 \\
\hline Citigrong & Banca & 95 & 256 \\
\hline
\end{tabular}

A manera de referencia, el PBI en América Latina 2004 en miles de Millones de US $\$$ fue: en México $(658,2)$, Brasil $(578,2)$, Venezuela $(103,5)$, Chile $(89,5)$ y Perú $(63,7)$. Del análisis de las dimensiones, se puede determinar que, si Perú quisiera comprar los activos de General Electric que ascienden a 329 mil millones de dólares, necesitariamos destinar el integro del PBI peruano durante cinco años y dos meses; para comprar Exxon Mobil cuatro años y cuatro meses. Es importante reflexionar sobre estas cifras para tener una aproximación de que significamos ante el mundo en el actual contexto de la globalización.

Otra tendencia de las finanzas en los últimos años, se presenta por la evolución social y económica de las necesidades de financiamiento de los agentes económicos, el cual motiva el desarrollo de nuevos instrumentos financieros o el mejoramiento de los instrumentos financieros actuales, este proceso es conocido como Innovación Financiera. En el Perú, observamos que los servicios financieros son parte del mercado globalizado. Muchas empresas peruanas han trascendido las fronteras del Perú. Por ejemplo, los eurobonos de Gloria S.A. y los ADR's (American Depositary Receipts) de Minas Buena Ventura S.A.

Otro aspecto importante es que la nueva economía, ha generado nuevas empresas donde el nuevo valor económico se genera a través de activos intangibles, y donde lo central es el flujo de información y la tecnología, las oportunidades de crear negocios son infinitos; lo cual coincide con lo señalado por Carlos Aimar (2000, p. 56) quien señala que «el manejo de los activos físicos ofreció en el pasado rendimientos ciertos y limitados. Los activos intangibles por el contrario ofrecen rendimientos inciertos y menos limitados». 
Por lo expuesto, hemos podido identificar el impacto de la globalización en las finanzas, debemos tener presente que los próximos años también serán años de cambio, donde lo central será la capacidad que deben tener los países y las empresas para su adaptación al cambio y aprovechamiento de oportunidades para aumentar el valor de las empresas. Antes de concluir, es importante señalar que las finanzas en el nuevo milenio deberán contar con nuevos enfoques para medir la creación de valor en las empresas.

\section{LA GESTIÓN EMPRESARIAL Y LAS MEDICIONES FINANCIERAS}

En la gestión de empresas, lo esencial es la utilidad. Ésta es mi opinión, una aseveración inadecuada. El objetivo de maximizar las utilidades es muy vago y genera varias interrogantes, tales como: ¿Son utilidades contables (valor de libros)?, ¿'son utilidades económicas (valor de mercado)?, ¿tiene algún peso la capacidad que deben tener las empresas de generar utilidades a largo plazo?, etc. La maximización de utilidades ignora el riesgo de distintas alternativas y el momento de la obtención de rendimientos (el valor del dinero en el tiempo), y además no toma en cuenta los flujos de efectivo. Debido a que el rendimiento y el riesgo son los factores fundamentales que determinan el precio de las acciones.

Tradicionalmente, las empresas miden la gestión financiera con indicadores financieros basados en la utilidad contable. Sin embargo, éste modelo en su determinación, no incluye el costo de oportunidad de los Accionistas por su capital invertido. La gestión de las finanzas en las empresas debe orientarse a incrementar la maximización de la riqueza de los accionistas, efectuar decisiones de inversión óptima y la maximización del precio de las acciones.

Al respecto, Lawrence J. Gitman (2000, pp. 15:18) señala: «el objetivo de la empresa, y por tanto de todos los gerentes y empleados, consiste en maximizar la riqueza de los propietarios para quienes operan. La riqueza de los propietarios corporativos se determina por medio del precio de una acción del capital social, que a su vez se basa en el momento de la obtención de rendimientos (flujos de efectivo), en su magnitud y en su riesgo". Es en ese sentido que los gerentes de finanzas solo deben tomar decisiones dirigidas al aumento del precio de las acciones. El profesor Gitman, adicionalmente, señala que «debido a que el precio de las acciones representa la riqueza de los propietarios de la empresa; la maximización del precio de las acciones significa la maximización de la riqueza de los propietarios». Refiere que los aspectos importantes relacionados con la maximización del precio de las acciones son: el Valor Económico Agregado (VEA), (del inglés economic value added, EVA), y la atención en los agentes relacionados».

El Valor Económico Agregado es una medida que muchas empresas utilizan para determinar si una inversión contribuye a maximizar la riqueza de los propietarios. Las acciones que realizan los gerentes de finanzas para maximizar la riqueza deben ser coherentes con la conservación de la riqueza de los agentes relacionados, grupos formado por accionistas, empleados, clientes, proveedores y acreedores que poseen un vínculo económico directo con la empresa. Es en ese sentido que una empresa que incluya en su modelo de gestión a los agentes 
relacionados, evita de manera consciente las acciones que los perjudican, como dañar su patrimonio a través de la transferencia de su riqueza a la empresa. El objetivo no consiste en maximizar el bienestar de los agentes relacionados, sino en conservarlo.

Con la globalización de las finanzas, y por lo expuesto, hoy en día cada vez más accionistas empiezan a involucrarse en el negocio, exigiendo a sus directores y gerentes un desempeño competitivo y la creación de valor para sus accionistas. Lo esencial es la creación de Valor. En creación de Valor lo que prima ya no son las utilidades de la empresa, ya que se puede generar utilidades descapitalizando la empresa a futuro o empleando programas de reducción de costos para conseguir beneficios contables, a expensas de crear valor para la empresa.

En ese sentido, los Accionistas esperan que sus directores y gerentes tomen decisiones que permitan la Creación de Valor; y este concepto, cada vez más, se convierte hoy en día en un tema central en la formulación de objetivos y estrategias empresariales. Al respecto, el Profesor Oriol Amat (2000, pp. 11:14) señala que «las empresas tratan de lograr diversos objetivos» entre los que se destacan:

- Aumentar el valor de la empresa, obteniendo la máxima utilidad con la mínima inversión de los accionistas y, además, obteniendo el Mínimo Costo Promedio de Capital.

- Administrar con el mínimo riesgo o riesgo aceptable, buscando una proporción adecuada entre el financiamiento de capital de terceros y la del capital de accionistas.

- Disponer de niveles adecuados de liquidez, mediante un financiamiento adecuado del capital de trabajo y buscando la mejor posición en el ciclo del efectivo de la empresa.

\section{MARCO CONCEPTUAL DEL} VALOR ECONÓMICO AGREGADO

\subsection{Significación del Valor Económico} Agregado

El concepto del Valor Económico Agregado, es hoy un tema actual, producto de la investigación realizada en 1980 por la Consultora Americana Stem Stewart \& Company, que desarrolló una metodología sobre el tema y hoy es utilizada por Compañías como Coca-Cola, General Electric, Microsoft, IBM, entre otras. Compañías como éstas lo utilizan para evaluar el rendimiento de las operaciones, los presupuestos y costos de capital, el nivel del capital de trabajo, la remuneración y compensación de sus más altos ejecutivos.

El Valor Económico Agregado constituye una herramienta gerencial clave para la planificación y el control estratégico; la cuantificación de la riqueza generada en una empresa; la gestión de las utilidades y los activos; la toma de decisiones y el control de gestión; la evaluación de desempeño por Unidades Estratégicas de Negocios.

El Valor Económico Agregado genera que los Directivos y gerentes piensen y actúen como si fueran los dueños del negocio, porque buscan ambos el mismo objetivo de maximizar el Valor de los Accionistas y se constituye en una herramienta importante para motivar y evaluar el rendimiento gerencial.

\subsection{Conceptos del Valor Económico Agregado}

Para identificar los conceptos centrales de Valor Económico Agregado, 
menciono a Oriol Amat (2000, pp. 12 y 13), quien señala: «el Valor Económico Agregado es una herramienta que permite calcular y evaluar la riqueza generada por la empresa, teniendo en cuenta el nivel de riesgo con el que opera. Por tanto, se trata de un indicador orientado a la integración puesto que considera los objetivos principales de la empresay. Asimismo, el mismo Amat (2000, p. 32) señala: «el Valor Económico Agregado es el importe remanente que queda una vez que se han deducido, de los ingresos, la totalidad de los gastos, impuestos y el costo de oportunidad de capital de los recursos externos y propios que se han invertido en los activos».

Otra concepción similar es la que plantea Carlos Cuevas (2001, pp. 13:22), donde precisa que «el Valor Económico Agregado es una medida de la utilidad producida sobre el costo de capitaly, precisando que el Valor Económico Agregado es aplicado al capital invertido y es calculado con base en utilidades después de impuestos. También es importante mencionar a $G$. Bennett Stewart, III (2000, p. 217) quien señala, «el Valor Económico Añadido, es la medida o indicador interno de la actuación operativa que refleja mejor el éxito de empresas a la hora de añadir valor a las inversiones de sus accionistas. El Valor Económico Añadido es el beneficio residual que queda de los beneficios de explotación después de que se ha deducido el costo de capitabs.

Por lo expuesto, debo señalar que el Valor Económico Agregado tiene por función reconocer el rendimiento general deduciendo todos los costos de capital externo y propios que se han invertido en activos de la empresa. En consecuencia, el Valor Económico Agregado es positivo, cuando la empresa ha generado una rentabilidad que supera el Costo de Oportunidad de los Accionistas; y por lo tanto, se crea Valor. Cuando el Valor Económico Agregado es negativo, significa que la empresa no ha generado una rentabilidad que supere el Costo de Capital de los Accionistas, y por lo tanto se está destruyendo Valor.

\subsection{Determinación del Valor Econó- mico Agregado}

Para determinar el Valor Económico Agregado se procede de la siguiente manera:

$\mathrm{EVA}=\mathrm{UAIDI}-\mathrm{ATN} \times \mathrm{CCPP}$

Donde:

UAIDI: Utulidades Ordinarias Antes de Intereses y Después de Impuestos. ATN: Activos Totales Netos CCPP: Costo de Capital Promedio Ponderado.

\section{Utilidades Ordinarias antes de Intereses} y después de Impuestos (UAIDI)

- Las utilidades operativas se determina solamente a partır de los resultados de las actividades ordinarias. Este resultado se calcula a partir de las Utilidades Netas, incorporando los gastos financieros y restando (o sumando) los beneficios (o las pérdidas) extraordinarios.

- Asimismo, se debe identificar las partidas contables que se deben ajustar para que el resultado sea lo más cercano a la realidad económica.

\section{Activo Total Neto (ATN)}

- El Activo Total Neto es el monto que se encuentra invertido en capital de trabajo, inmuebles, equipos, tecnología y otras inversiones. En la determinación de los activos a considerarpueden serlos existentes al inicio, al final del ejercicio o un promedio. 
- Los Valores de los Activos deben ser los Valores de Mercado, a fin de cuantificar correctamente los recursos comprometidos en la empresa, de existir diferencia significativa entre los Valores Contables y Valores de Mercado se puede reconocer un Ajuste por Plusvalía o Minusvalía de Activos.

- Se debe deducir del valor de los Activos la financiación automática sin costo explicito que aportan los denominados Pasivos Espontáneos (Estado, proveedores, seguridad social, remuneraciones por pagar, otras cuentas por pagar).

\section{Costo de Capital Promedio Ponderado (CCPP)}

- El Costo de Capital Promedio se refiere al costo promedio de toda la financiación utilizada que ha obtenido la empresa, provenientes de pasivos con costo explícito y de capital propio.

- Para calcular el Costo de las Deudas, se debe efectuar desde la perspectiva de los accionistas $y$, por lo tanto, tiene que ser después de impuestos. Al utilizar estos costos, se tiene en cuenta el riesgo de los recursos financieros utilizados.

- Para calcular el Costo de los Fondos Propios, éste debe estar en función del Costo de Oportunidad de los Accionistas. Este costo se calcula a partir del tipo de interés de mercado para las inversiones sin riesgo o de riesgo muy bajo, al que se añade una prima por riesgo en función de las características de la empresa y del sector económico.

\subsection{E1 Objetivo de la Empresa: Valor Económico Agregado}

El Objetivo de toda empresa es generar valor económico para sus accionistas de manera creciente y sostenida en el tiempo; lo cual implica que en el largo plazo el valor económico de la empresa siga creciendo. Lo anterior significa desterrar el concepto de utilidad como indicador de éxito para los objetivos empresariales. A continuación se presenta un ejemplo para precisar que se entiende por Valor Económico Agregado.

Supongamos que la empresa Alfa ha generado en el año 2004 una utilidad de US $\$ 30$ millones, habiendo invertido US $\$ 200$ millones en capital expuesto en caja y bancos, cuentas por cobrar, existencias y activos fijo neto. Si a la empresa le cuesta el capital $12 \%$ anual (US $\$ 24$ millones), entonces la empresa ha generado un Valor Económico de US $\$ 6$ millones. Por otra parte, la empresa Beta ha generado en el año 2004 una utilidad de US $\$ 40$ millones, habiendo invertido US $\$ 400$ millones de capital invertido. Si a la empresa le cuesta el capital $12 \%$ anual (US $\$ 48$ millones), puede decirse que aún cuando la empresa Beta haya generado utilidad, ésta empresa destruye valor económico.

Lo anterior nos permite señalar que generar utilidad no implica producir valor económico; hay que tomar en cuenta el capital invertido en lograr esta utilidad; y una mayor utilidad tampoco significa mayor valor. En el ejemplo anterior se ha determinado que la compañía Alfa ha generado menor utilidad, pero ha generado más valor económico por utilizar mucho menos capital en la inversión.

En conclusión, para que una empresa sea exitosa, no basta con evaluar el nivel de utilidad generado; es necesario comparar dicha utilidad con el costo de capital invertido, para generarla. En consecuencia, solo se crea valor económico cuando la utilidad es superior al costo del capital invertido en generarla. 
SUPUESTOS

EMPRESA ALFA

- Utilidad $=30 \mathrm{M}$

- Capital

invertido $=200 \mathrm{M}$

- Costo del

capital $=12 \%$

\section{EMPRESA BETA}

$$
\begin{array}{ll}
\text { Utilidad } & =40 \mathrm{M} \\
\begin{array}{l}
\text { Capital } \\
\text { invertido }
\end{array} & =400 \mathrm{M} \\
\text { Costo del } & \\
\text { capital } & =12 \%
\end{array}
$$

EMPRESA ALFA

30

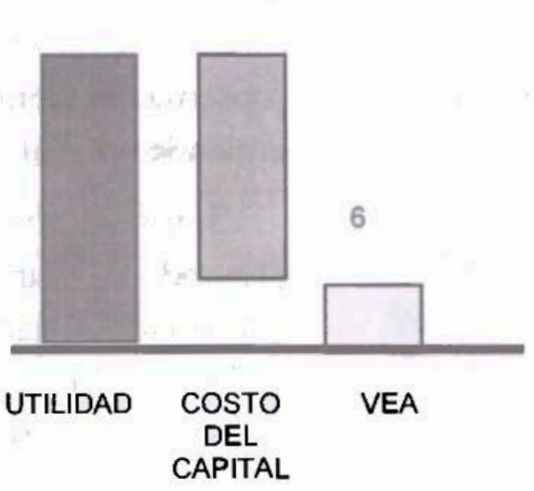

EMPRESA BETA
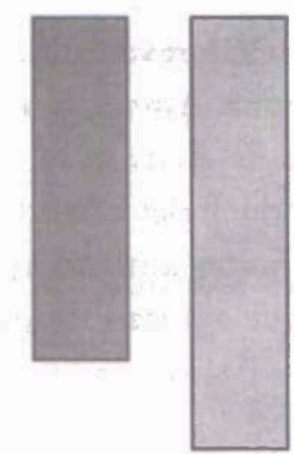

(8)

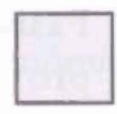

VEA

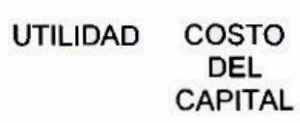

\section{LAS FINANZAS Y LAS ESTRA- TEGIAS EMPRESARIALES}

Como se menciono anteriormente, en el contexto de la globalización, en donde existe una mayor competitividad, las empresas tienen que desarrollar nuevas herramientas en el campo de las finanzas. Es así que surgen los nuevos enfoques de las finanzas: Creación de Valor y Valor Económico Agregado. La Creación de Valor vincula las estrategias empresariales y las finanzas; toda vez que en la actualidad, los modelos de negocios y los planes estratégicos están basados en el modelo de valor que proporciona un enfoque de visión de futuro. En el modelo de valor, el valor empresarial es creado por la red de valor del accionista. Al respecto, Alejandro Indacochea (2000, pp. 97:104) señala: «en la red de valor del accionista hablamos de los impulsores de valor, es decir, los factores que afectan la creación de valor del negocio: vida útil, crecimiento de ventas, inversiones, márgenes de utilidad operativa, todos los componentes del flujo de caja libre. La gerencia se aboca a una gerencia de creación de valory. En ese sentido, todas las decisiones gerenciales vinculadas a los procesos de recomposición empresariales: reestructuración, rediseños, alianzas estratégicas, fusiones, joint venture, se tienen que hacer bajo el enfoque de creación de valor.

Un aspecto importante en las finanzas del ValorEconómico Agregado es identificar las variables que influyen en la generación del valor de la empresa; de tal forma que se pueda actuar directamente sobre las variables como ingresos, costos, gastos de capital, que puedan tener una influencia directa en la generación de valor; y adicionalmente, es que a través de estos impulsores de valor se puedan establecer estrategias empresariales que afecten de manera directa en la generación de valor maximizando, con ello, el valor de los accionistas.

El enfoque de creación de valor constituye el cambio más importante en las finanzas modernas, la integración de las finanzas con la estrategia es un hecho y la nueva gestión empresarial tiene como 
objetivo crear valor en la organización y no dedicarse únicamente a la reducción de costos del pasado, eso no crea valor en las organizaciones de la empresa.

\section{ESTRATEGIAS FINANCIERAS PARA AUMENTAR EL VALOR ECONÓMICO AGREGADO}

Existen cuatro estrategias financieras básicas para incrementar el Valor Económico Agregado de una empresa o una unidad de negocio: aumentar las utilidades brutas con los activos existentes; disminuir los gastos operativos; reducir el capital invertido, manteniendo las utilidades, e invertir solo en activos con rentabilidad superior al costo de capital y reducir el costo de capital. Con alguna de estas estrategias o actuando en conjunto, es posible aumentar el Valor Económico Agregado y, porlo tanto, el valor creado por la empresa para el accionista.

\subsection{Estrategias para Aumentar la Utilidad Bruta}

Las acciones que se deben realizar para aumentar las utilidades brutas, con los activos existentes, son básicamente aumentando los ingresos por ventas y disminuyendo los costos de producción y/ o servicio.

\subsubsection{Estrategias para Aumentar los Ingresos por Ventas}

Las estrategias que se pueden utilizar para el logro de este objetivo financiero, entre otras son:

- Aumentar el número de productos vendidos y/o servicios prestados.

- Mejorar los precios de nuestros productos y/o servicios, sujeto a la aceptación del mercado.
- Desarrollar nuevos mercados para nuestros productos y/o servicios actuales.

- Desarrollar un modelo de gestión orientado hacia el cliente, es decir, desarrollar productos y/o servicios con valor agregado para los clientes.

- Identificar necesidades que los clientes buscan satisfacer para poder atenderlos con el mejoramiento de productos y/o servicios o el desarrollo de nuevos productos y/o servicios a través de procesos de innovación.

- Aumentar el volumen de ventas de los productos y/o servicios que generan mayores rentabilidades brutas con respecto al portafolio de productos, $y$, en su caso, descontinuar productos $\mathrm{y} / \mathrm{o}$ servicios que no generen rentabilidades brutas a la organización, logrando un aumento del margen bruto promedio.

- Revisar el sistema de precios (precio justo - margen), donde el margen debe fijarse en base a estudios especiales de los costos y los precios de la competencia.

- Mejorar los servicios de atención al cliente, incluyendo la post venta y/o post servicio que se requieran, mejorar la comunicación y fidelidad de los clientes.

\subsubsection{Estrategias para Disminuir los Costos de Producción y/o Servicio}

Las estrategias que se pueden utilizar para el logro de este objetivo financiero son:

- Desarrollar un modelo de gestión de costos orientado hacia la búsqueda permanente de factores de producción y/o servicios más económicos:

- Disminuir los costos fijos por unidad de producto $y / o$ servicio como consecuencia del aumento de los ingresos por ventas. 
- Obtener descuentos adicionales en la adquisición de bienes materiales $\mathrm{y} / \mathrm{o}$ servicios, a través de negociación directa, compras corporativas, compras asociadas con organizaciones del sector, por efecto de la continuidad volumen negociado, etc.

- Identificar ahorros en costos a través de sistemas de costos adecuados, rediseño de procesos de producción para que cuesten menos, sin afectar la calidad del mismo.

- Reconvertir costos fijos en costos variables especialmente en nómina, para que la empresa pueda manejar los recursos financieros con razonable flexibilidad.

- Tercerizar (outsourcing), es decir, subcontratar aquellos procesos en los que la empresa no sea competitiva, y no agreguen valor al cliente, según sea el caso.

- Optimizar los activos para disminuir los gastos vinculados con los mismos. Por ejemplo, Si se reduce el nivel de stock de las existencias en una industria, pueden reducirse los costos vinculados al almacenaje.

- Incrementar la productividad del personal a través de capacitación, motivación, incentivos, etc. en las tareas del desarrollo de la producción y/o servicio.

\subsection{Estrategias para disminuir los gastos operacionales}

Las acciones que se deben realizar para reducir los gastos operativos son básicamente las siguientes:

- Desarrollar un modelo de gestión por cada una de las áreas operacionales de la organización, orientado hacia la eficiencia y eficacia.
- Identificar los procesos críticos en la gestión, establecer las tareas involucradas y determinar los recursos humanos materiales requeridos para realizar cada actividad de manera óptima, de tal forma que se puedan reducir los costos innecesarios.

- Disminuir los gastos fijos por unidad de producto y/o servicio como consecuencia del aumento de los ingresos por ventas.

- Reconvertir gastos fijos en gastos variables, especialmente en contratación de la nómina de la fuerza de ventas y personal de cobranza de la organización.

- Tercerizar (outsourcing). Esta acción es central en la parte operativa de la empresa, en lo que se debe tener en cuenta lo mencionado anteriormente sobre éste tema.

- Utilizar la tecnología de información y comunicación para disminuir los gastos de papelería, impresos, comunicación, etc.

- Incrementar la productividad del personal a través de capacitación, motivación, incentivos, etc. en las tareas del desarrollo de la gestión operativa de la organización.

- Reducir el gasto anual de impuesto a la renta, mediante una adecuada planificación tributaria que permita identificar oportunidades que ofrece la legislación tributaria vigente (deducciones de renta, desgravaciones fiscales, métodos alternativos de imposición, etc.).

\subsection{Estrategias para Reducir el Capital Invertido}

Las acciones que se deben realizar para reducir el capital invertido existente (activos totales netos), manteniendo las utilidades son básicamente los siguientes: 
- Reducir el nivel del efectivo, mediante técnicas de gestión de tesorería y planeamiento financiero. Por ejemplo: reducir los días del ciclo de efectivo.

- Reducir las cuentas por cobrar, mediante técnicas de gestión de crédito. Por ejemplo: otorgar descuentos por pronto pago de las cuentas pendientes, seleccionar los mecanismos de cobro más adecuados, usar la venta de cartera, analizar los créditos vencidos y por vencer, implementar incentivos para el personal sobre la base de ventas cobradas, recuperar los créditos incobrables, etc.

- Reducir el nivel de existencias, mediante técnicas de gestión de inventarios. Por ejemplo: reducir el ciclo de producción y abastecimiento, producir en base a pedidos de clientes, simplificar productos a través de producir solo productos que utilicen los mismos insumos, aplicar el modelo de inventarios justo a tiempo, disminuir el número de productos producidos en condición de fallados, irregulares o para reprocesos de producción.

- Reducir activos fijos, mediante técnicas de gestión de inversiones fijas. Por ejemplo: liquidar o vender activos fijos obsoletos, o improductivos, alquilar activos fijos en lugar de comprar la propiedad de los mismos, tercerizar procesos para poder eliminar los activos correspondientes, efectuar estudios para determinar la capacidad de planta o infraestructura óptima.

\subsection{Estrategias para reducir el costo de capital}

Las acciones que se deben realizar para reducir el costo promedio ponderado del capital, son básicamente las siguientes:
- Incrementar el apalancamiento financiero, si el rendimiento de los activos es mayor al costo de la deuda, hasta un nivel aceptable de riesgo y tenga efectos positivos para la organización.

- Obtener menores costos promedios de deudas, lo cual se puede conseguir buscando tasas de interés corporativas o preferenciales en los bancos del mismo grupo empresarial, record crediticio de la organización, etc.

- Mejorar la estructura financiera de acuerdo a la disponibilidad de líneas de financiamiento y al énfasis en la reducción del riesgo de la empresa.

- Buscar nuevas fuentes de financiación con menores costos de financiación; por ejemplo: emisión de bonos corporativos, avance en cuenta, etc.

\section{INVESTIGACIÓN APLICADA: EL CASO DE AMERICAN TEXTIL S.A.}

\subsection{Antecedentes}

A continuación, se presenta una investigación aplicada American Textil S.A.; empresa peruana que ocupa el décimo lugar en la industria textil de Jeans que atiende el mercado nacional.

Para determinar el Valor Económico Agregado, presentamos el Balance General y el Estado de Ganancias y Pérdidas correspondiente al año 2003 de la empresa real American Textil S.A. y que se detalla en los Anexos Nros. 1 y 2 , respectivamente. Es importante señalar que la empresa American Textil S.A. mantiene una tasa de préstamos bancarios de $15 \%$ anual, un costo de oportunidad de accionistas de $23 \%$ anual y una tasa impositiva de renta del $30 \%$ anual. 
Anexo N."1

AMERICAN TEXTIL S.A.

Balance General al 31 de diciembre de 2003

(expresado en miles de dólares)

$\begin{array}{lc}\text { Activo Importe } & \\ \text { Caja y bancos } & 1373 \\ \text { Cuentas por cobrar } & 4627 \\ \text { Existencias } & 5000 \\ \text { Activo fijo } & \underline{14000} \\ & 25000 \\ & ===== \\ \text { Pasivoy Patrimonio } & \\ \text { Impuestos y provecdores } & 3000 \\ \text { Deuda bancaria } & 8000 \\ \text { Capital } & 12000 \\ \text { Utilidad del ejercicio } & 2000 \\ & 25000\end{array}$

Como se puede observar, durante el año 2003, la empresa ha generado utilidades por US $\$ 2,000$ miles que representa solo el $5 \%$ de la venta anual y que utilizaba una inversión de Capital de US $\$ 22,000$ miles (cifra que equivale a 11 veces la utilidad). Asimismo, considerando que el costo de oportunidad de accionistas es del 23\% anual; la empresa American Textil S.A., a pesar de generar utilidad, destruía Valor Económico Agregado. El Anexo N. ${ }^{\circ} 3$ presenta la determinación el Costo Promedio Ponderado de Capital correspondiente al año 2003. En su determinación, el costo anual de intereses al ser gasto con efecto impositivo se aplica la tasa del impuesto a la renta del $30 \%$ al costo anual.

Para efectos de determinar si esta empresa generaba o no generaba valor, se ha emitido el Anexo N. ${ }^{\circ} 4$, Reporte Financiero de Valor Económico Agregado antes de la reestructuración. Tal como se puede apreciar, el Valor Económico Agregado es negativo en US $\$ 1960$ miles, por lo que se aprecia un resultado de gestión que no hagenerado valor para sus accionistas.

\subsection{Objetivos}

Para efectos de lograr el mejoramiento del Valor Económico Agregado, la empresa
$\operatorname{Anexo} N .{ }^{2} 2$

AMERICAN TEXTIL S.A

Estado de Ganancias y Pérdidas en el año 2003 (expresado en miles de dólares)

\begin{tabular}{lr} 
Importe & \\
\hline Ventas & 40000 \\
Costo de ventas & 30000 \\
Utilidad bruta & 10000 \\
Gastos operativos & 7140 \\
\cline { 2 - 2 } Utilidad operativa & 2860 \\
Gastos financieros & 1200 \\
ingresos extraordinarios & 1800 \\
Gastos extraordinarios & 600 \\
Utilidad antes de impuestos & 2860 \\
Impuesto a la Renta & 860 \\
Utilidad neta & 2000
\end{tabular}

American Textil S.A. decidió la reestructuración total de las operaciones de la empresa, con el objetivo de incrementar, de manera significativa, el Valor Económico Agregado, por lo que esta investigación permite comprender el proceso seguido en la implementación de estrategias para el mejoramientode ValorEconómico Agregado.

\subsection{Desarrollo de la investigación}

Para una mejor orientación del proceso seguido en el desarrollo de las acciones que se realizaron, el Anexo N. ${ }^{\circ} 5$ presenta el Árbol de Valor de la empresa American Textil S.A., para representar gráficamente y en forma analítica el Valor Económico Agregado antes de la reestructuración.

En el Árbol de Valor presentado en el Anexo N. 5 se aprecia que el Valor Económico Agregado tiene dos grandes componentes y cinco ejes, por lo que el incremento del Valor Económico Agregado de American Textil S.A. se desarrolló con acciones simultáneas, seleccionando y ejecutando las siguientes cinco estrategias financieras: aumentar las ventas, mejorar la rentabilidad bruta, reducir los gastos operativos, reducir el capital invertido y reducir el costo de capital. 
Anexo N.0 3

AMERICAN TEXTIL S.A.

Costo Promedio Ponderado de Capital

Al 31 de diciembre de 2003

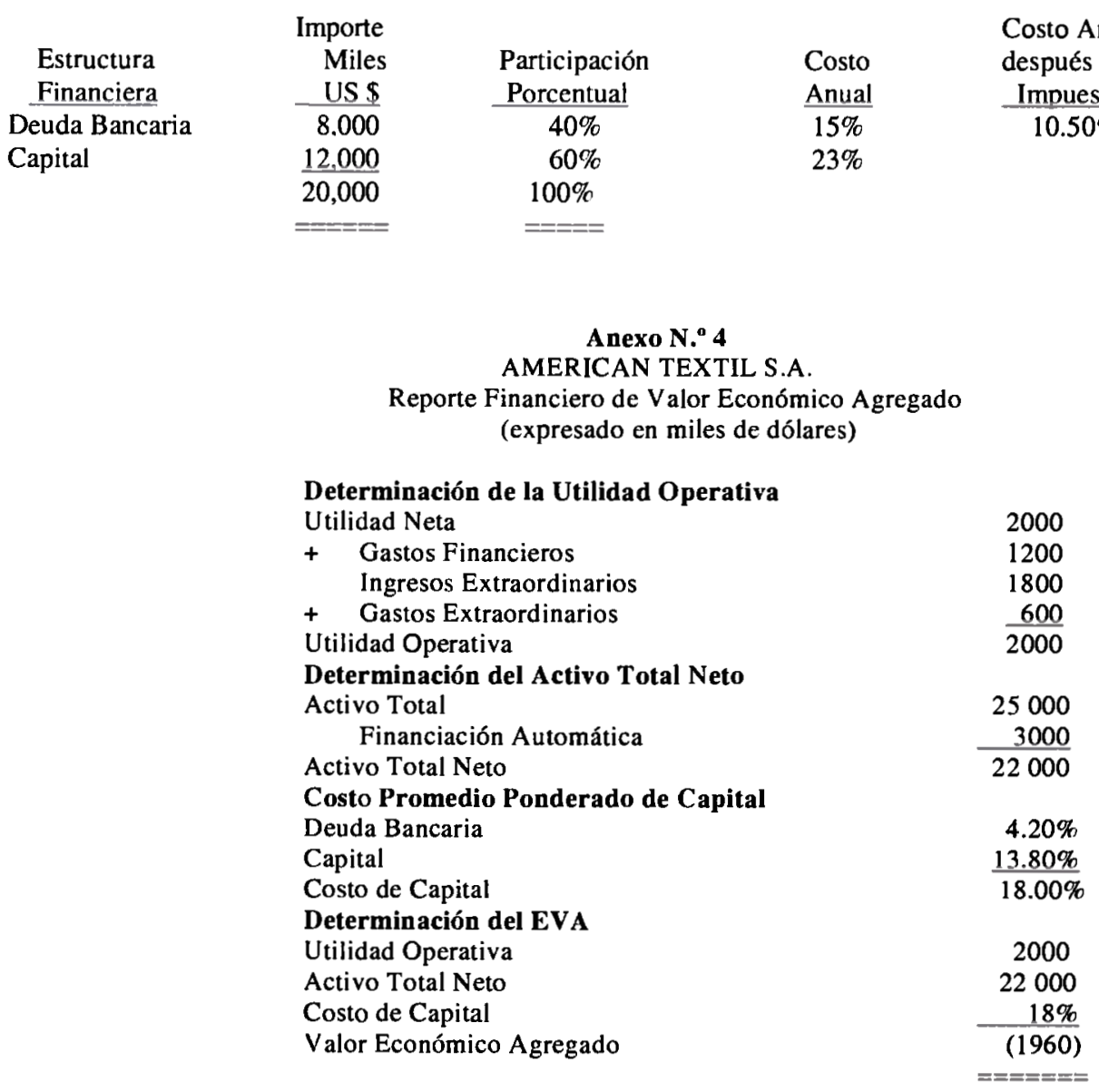

\section{Anexo N. ${ }^{\circ} 5$}

AMERICAN TEXTIL S.A.

El Árbol de Valor antes de la Reestructuración (expresado en miles de dólares)

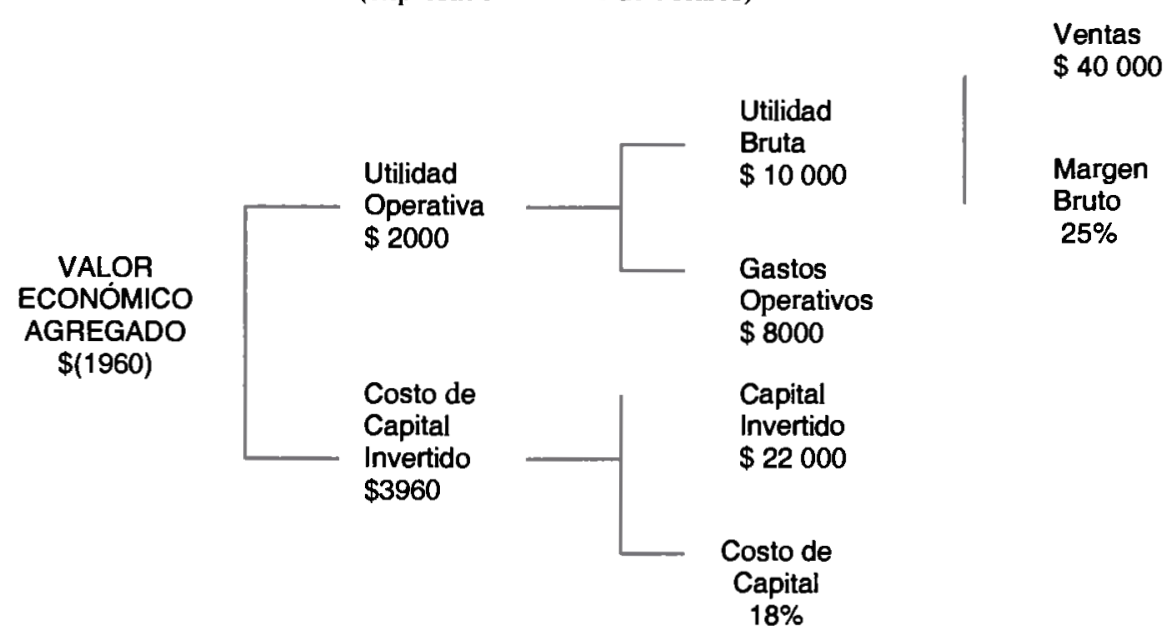


Aumentar las ventas. Para aumentar las ventas existen dos formas de lograrlo. La primera, aumentando la cantidad de unidades vendidas; y la segunda, aumentando el valor de venta unitario. Esta última opción no se ejecutó por la posición competitiva de la empresa. La empresa American Textil S.A. decidió aumentar las unidades vendidas en $10 \%$ en todos sus productos y desarrolló un plan de marketing para incrementar la venta a través de clientes actuales y conseguir nuevos clientes. Para efectos de determinar el impacto financiero por aumento de las ventas, se presenta el Anexo N. ${ }^{\circ}$ 6, Reporte de Ventas antes y después de la reestructuración. Este último incluye el efecto de eliminar dos productos. Lo anterior generó un aumento en los ingresos anuales de American Textil, alcanzado un nivel de US $\$ 43746$ miles.

Mejorar la Rentabilidad Bruta. Para mejorar la rentabilidad bruta, se hicieron esfuerzos para reducir el costo de ventas y optimizar el portafolio de productos. Con respecto a la reducción de los costos, se efectuó un proceso de negociación directa con los proveedores de telas de materias primas, para obtener descuentos adicionales. Producto de esta negociación favorable, se logró un impacto en los costox variables unitarios reduciéndolos en $10^{\circ}$ sobre toda la línea de productos. Otr: esfuerzo importante que se realizó fue la identificación de oportunidades de ahorr: de costos fijos por mejora de procesos logísticos, lo cual significó un ahorro de Lis $\$ 272$ miles. Lo anterior se visualiza en ed Anexo N. ${ }^{\circ} 7$, Reporte de Costos Anuales antes y después de la reestructuración, éste último incluye el efecto de eliminar dos productos de la producción.

Como se puede apreciar, si bien es cierto el costo total antes de la reestructuración ascendía a US $\$ 30000$ miles, y después de la reestructuración asciende a US $\$ 29310$ miles; este menor costo, se debe también al incremento del $10 \%$ de las unidades producidas por efecto del aumento en el nivel de unidades vendidas. Por otro lado, para efectos de optimizar el portafolio de productos se logró eliminar del portafolio dos productos que tenían muy bajos márgenes y seguramente no generaban valor. Tal como se puede apreciar en el Anexo N. ${ }^{\circ}$, Reporte de Rentabilidad Bruta, la empresa American Textil S.A. pasó de un margen bruto promedio de $25 \%$ a un margen bruto promedio de $33 \%$, después de la reestructuración.

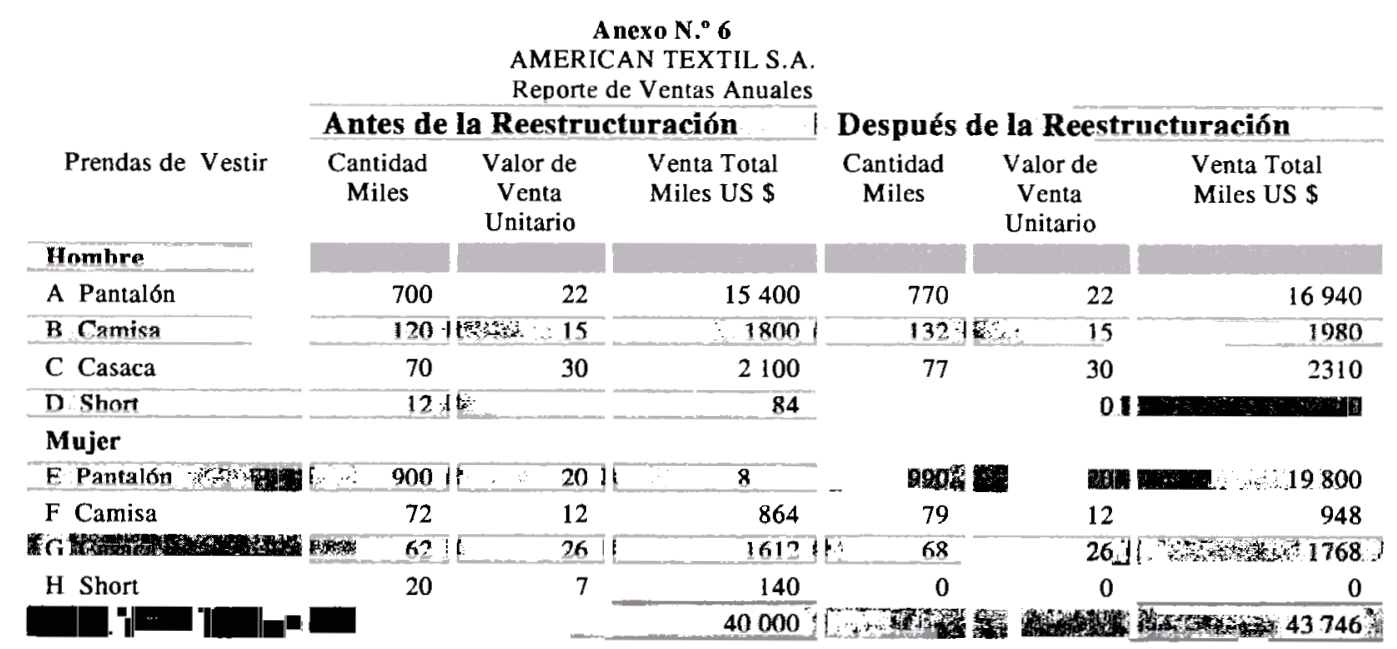




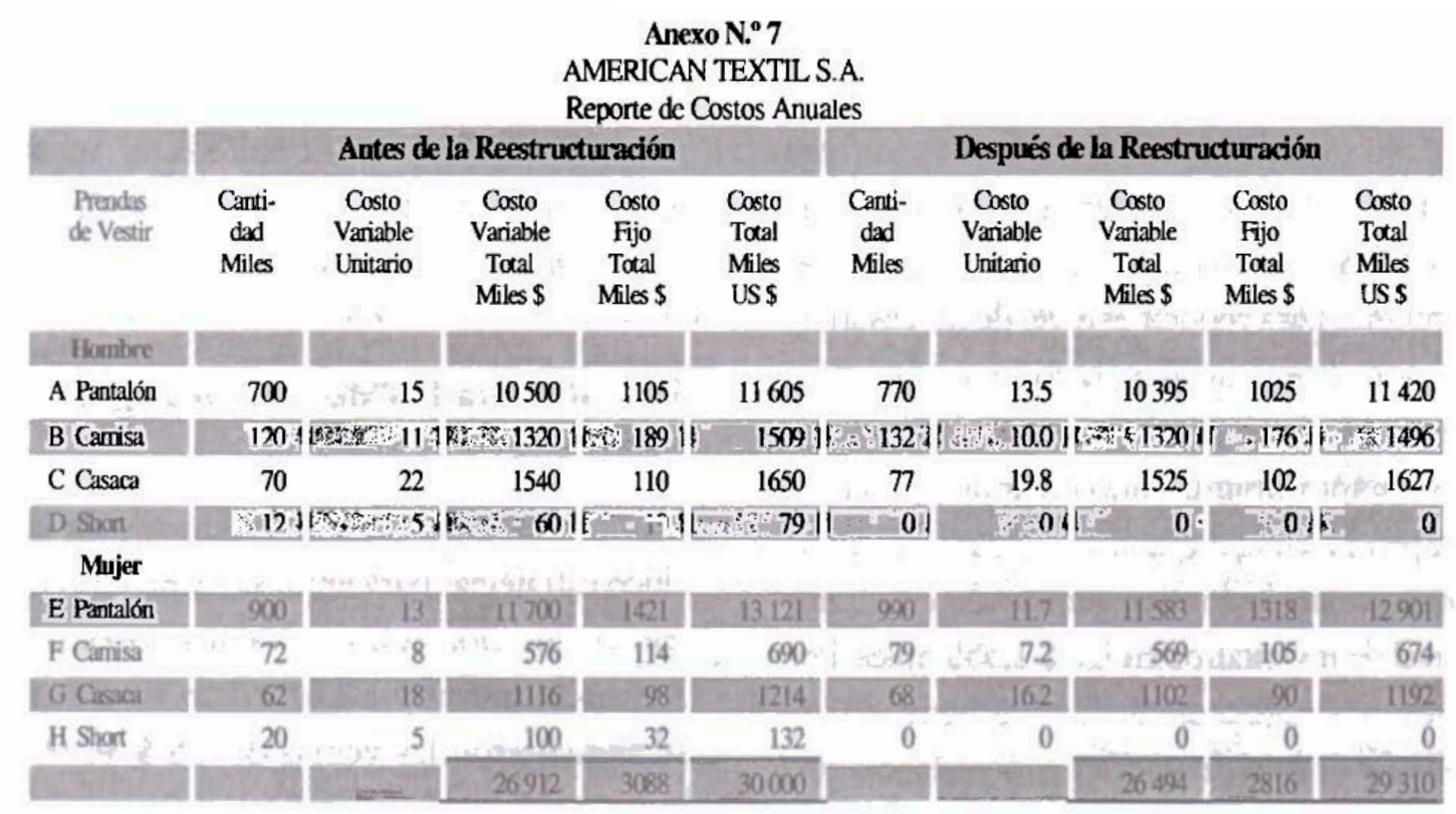

\begin{tabular}{|c|c|c|c|c|c|c|c|c|}
\hline \multicolumn{9}{|c|}{$\begin{array}{c}\text { Anexo N. } \mathbf{0}^{\mathbf{8}} \\
\text { AMERICAN TEXTIL S.A. } \\
\text { Reporte de Rentabilidad Bruta }\end{array}$} \\
\hline & \multicolumn{4}{|c|}{ Antes de la Reestructuración } & \multicolumn{4}{|c|}{ Después de la Reestructuración } \\
\hline $\begin{array}{l}\text { Prendas } \\
\text { de Vestir }\end{array}$ & $\begin{array}{l}\text { Venta } \\
\text { Total } \\
\text { Miles \$ }\end{array}$ & $\begin{array}{l}\text { Costo } \\
\text { Total } \\
\text { Miles } \$\end{array}$ & $\begin{array}{l}\text { Utilidad } \\
\text { Bruta } \\
\text { Miles \$ }\end{array}$ & $\begin{array}{l}\text { Rentabilidad } \\
\text { Bruta sobre } \\
\text { la Venta }\end{array}$ & $\begin{array}{l}\text { Venta } \\
\text { Total } \\
\text { Miles } \$\end{array}$ & $\begin{array}{l}\text { Costo } \\
\text { Total } \\
\text { Miles } \$\end{array}$ & $\begin{array}{l}\text { Utilidad } \\
\text { Bruta } \\
\text { Miles \$ }\end{array}$ & $\begin{array}{l}\text { Rentabilidad } \\
\text { Bruta sobre } \\
\text { la Venta }\end{array}$ \\
\hline \multicolumn{9}{|l|}{ Hombre } \\
\hline A Pantalón & 15400 & 11605 & 3795 & $24.64 \%$ & 16940 & 11420 & 5520 & $32.59 \%$ \\
\hline B Camisa $=11$ & 1800 & 15097 & $32: 29111$ & $16.16 \%$ & 1980 & 50.1496 & 484 & $24.44 \%$ \\
\hline C Casaca & 2100 & 1650 & 450 & $21.43 \%$ & 2310 & 1627 & 683 & $29.57 \%$ \\
\hline D Short & 84 & 79 & 5 & $5.95 \%$ & $01 !$ & 0 & 0 & 0 \\
\hline \multicolumn{9}{|l|}{ Mujer } \\
\hline E Pantalón & 18000 & 13121. & 4879 & $27.11 \% 11$ & 198011 & $12901 \%$ & 6899 & $34.84 \%$ \\
\hline F Carnisa & 864 & 690 & 174 & $20.13 \%$ & 948 & 674 & 274 & $28.90 \%$ \\
\hline G Cacaca & $\times 5161291$ & 121416 & (2): $\ln _{3} 398 \div$ & $24.68 \%$ & $17681 !$ & 1102 & 576 & (25) \\
\hline \multirow[t]{2}{*}{ H Short } & 140 & 132 & 8 & $5.71 \%$ & 0 & 0 & 0 & 0 \\
\hline & 40000 & 30000 & 10000 & $25.00 \%$ & 43746 & 29310 & 14436 & A. \\
\hline
\end{tabular}

Reducir los Gastos Operativos. Con respecto a los gastos operativos la empresa tenía gastos por US $\$ 8000$ miles incluido impuestos; lo cual significaba un $20 \%$ de las ventas, cifra elevada según benchmarking en empresas similares.

En este proceso se efectuó una mejora en la productividad de los procesos de apoyo administrativos, generando un ahorro de costos de US $\$ 700$ miles. Asimismo, se efectuó una reingeniería a los procesos administrativos, identificando gastos improductivos que se eliminaron por US $\$ 300$ miles, que impactaban la generación de valor. Como consecuencia de estas dos acciones, la empresa empezó a gestionar gastos operativos por US $\$ 7000$ miles, incluido impuestos que logro representar el $16 \%$ de las ventas.

Reducir el capital invertido. Para efectos de logra reducir el capital invertido que ascendia a US $\$ 22000$ miles, se desarrolló la compresión de activos.

Se empezó con reducir el nivel de cuentas por cobrar para cumplir los 30 días de crédito autorizado a nuestros clientes, se 
tuvo que acelerar cobranzas a través de una depuración de cuentas vencidas, generando un efecto de US $\$ 660$ miles y administrar el aumento de cartera por un aumento de ventas en US $\$ 371$ miles. También se efectuaron acciones para reducir el nivel de inventarios, donde el ciclo de inventarios era de 60 días, acelerando la reducción del ciclo de inventarios optimizando tiempos y procesos de producción, logrando alcanzar un ciclo de inventarios de 45 días, generando un menor nivel de inventarios en US $\$ 1,336$ miles. Por último, se procedió a eliminar activos fijos obsoletos y en desuso, en concordancia con el proceso de optimización de la planta, generando una disminución deUS $\$ 375$ miles.

Lo anterior, permitió una disminución de capital invertido en US $\$ 2000$ miles, determinándose un nivel de capital invertido de US $\$ 20000$ miles después de la reestructuración.

Reducir el Costo de Capital. Para reducir el costo de capital informado en el Anexo $\mathrm{N}^{\circ} 3$ y que ascendía a $18 \%$, se han efectuado dos acciones importantes: incrementar el apalancamiento y disminuir el costo promedio de la deuda bancaria.

Para incrementar el apalancamiento, se logró modificar la relación entre la deuda y patrimonio que era de 1 a 1.5 , obteniéndose un nivel mayor de deuda $y$ comprometer menos patrimonio, obteniéndose una relación de 1 a 1 . Asimismo, se logró reducir el costo promedio de la deuda bancaria en $1 \%$ (de15\% a $14 \%$ anual), por reducción de número de fuentes bancarias y negociación directa.

Las acciones anteriores han permitido reducir el costo de capital de $18 \%$ a $16.40 \%$. El Anexo N. ${ }^{\circ} 9$ presenta la determinación del costo de capital después de la reestructuración.
Asimismo, y a manera de concebir en forma resumida las estrategias financieras efectuadas en American Textil S.A., el Anexo N. ${ }^{\circ} 10$ presenta la Matriz de Gestión de Estrategias Financieras.

\subsection{Resultados de la Investigación Efectuada}

Producto de la implementación de las cinco estrategias para impulsar el proceso de reestructuración sobre el Valor Económico Agregado de la empresa American Textil S.A., se aumentaron las ventas de US $\$ 40000$ miles a US $\$ 43746$ miles, se mejoró la rentabilidad bruta de $25 \%$ a $33 \%$, los gastos operativos disminuyeron de US $\$ 8000$ miles a US $\$ 7000$ miles, se logró reducir el capital invertido de US $\$ 22000$ miles a US $\$ 20000$ miles y el costo de capital se redujo de $18 \%$ a $16.40 \%$. De esta manera, la empresa pasó de destruir Valor EconómicoUS $\$ 1960$ millones por año a generar US $\$ 4156$ millones por año, exclusivamente a través de un proceso de reestructuración financiera orientado a la eficiencia operativa y financiera.

El anexo 11 presenta el Árbol de Valor después de la reestructuración para visualizar el Valor Económico Agregado de US $\$ 4156$ millones con la cual la empresa American Textil S.A., pasó a ocupar el cuarto lugar de la industria textil de Jeans en el Perú.

Para concluir esta investigación aplicada a American Textil S.A., debo precisar que no hubiese sido posible lograr un aumento del Valor Económico Agregado, sin contar con una dirección y gerencia orientada hacia una cultura de valor, el rediseño de los sistemas gerenciales de información, la evaluación y control de estrategias y la política de incentivos a la administración por el logro del objetivo general de crear valor para el accionista. 
Anexo N." 9

AMERICAN TEXTIL S.A.

Costo Promedio Ponderado de Capital después de la Reestructuración

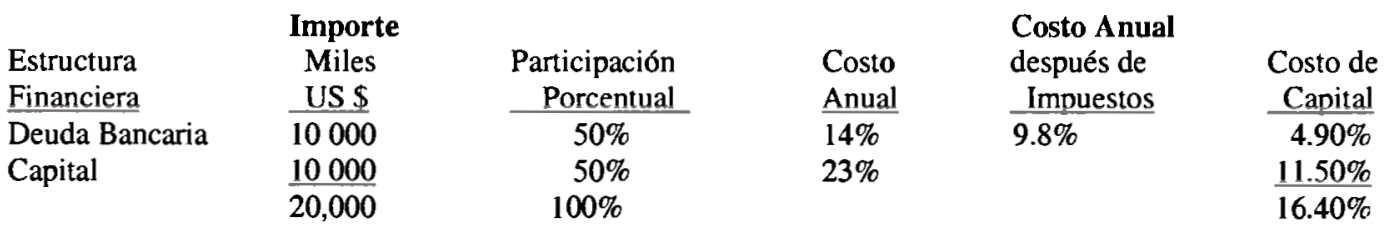

Anexo N.* 10

AMERICAN TEXTIL S.A.

Matriz de Gestión de Estrategias Financieras para incrementar el Valor Económico Agregado

\begin{tabular}{|c|c|c|c|}
\hline $\begin{array}{l}\text { Variables de } \\
\text { Desempeño }\end{array}$ & $\begin{array}{l}\text { Estrategias } \\
\text { Financieras }\end{array}$ & Objetivos Estratégicos & Acciones Estratégicas \\
\hline Ingresos & $\begin{array}{l}\text { Aumentar las ventas } \\
\text { con productos } \\
\text { actuales. }\end{array}$ & $\begin{array}{l}\text { - Aumentar las unidades } \\
\text { vendidas en } 10 \% \text {. }\end{array}$ & $\begin{array}{l}\text { - Desarrollar un plan de marketing, de } \\
\text { acuerdo a objetivos de ventas. }\end{array}$ \\
\hline Rentabilidad Bruta & $\begin{array}{l}\text { Mejorar la } \\
\text { Rentabilidad Bruta. }\end{array}$ & $\begin{array}{l}\text { - Mejorar el margen bruto } \\
\text { promedio en un } 33 \% \text {. }\end{array}$ & $\begin{array}{l}\text { - Reducir costos variables en } 10 \% \text {, producto } \\
\text { de la obtención de descuentos adicionales } \\
\text { en la adquisición de materias primas. } \\
\text { - Reducir el costo fijo en US } \$ 272 \text { miles } \\
\text { por mejora de procesos logísticos. } \\
\text { - Eliminar del portafolio de productos con } \\
\text { bajo margen. }\end{array}$ \\
\hline $\begin{array}{l}\text { Gastos } \\
\text { Operacionales }\end{array}$ & $\begin{array}{l}\text { Reducir los Gastos } \\
\text { Operativos. }\end{array}$ & $\begin{array}{l}\text { - Alcanzar una cifra de } \\
\text { gastos operacionales } \cdots \text { no } \\
\text { mayor al } 16 \% \text { de las ventas, } \\
\text { con una disminución de US } \\
\$ 1000 \text { miles. }\end{array}$ & $\begin{array}{l}\text { - Mejorar la productividad de los procesos } \\
\text { de apoyo para generar un ahorro de US } \$ \\
700 \text { miles. } \\
\text { - Eliminar gastos improductivos por US } \$ \\
300 \text { miles. }\end{array}$ \\
\hline Capital Invertido & $\begin{array}{l}\text { Reducir el Capital } \\
\text { Invertido. }\end{array}$ & $\begin{array}{l}\text { - Reducir nivel de cuentas } \\
\text { por cobrar a } 30 \text { días con un } \\
\text { efecto neto de US } \$ 289 \\
\text { miles. } \\
\text { - Reducir nivel de } \\
\text { inventarios de } 60 \text { a } 45 \text { días, } \\
\text { con un efecto de US } \$ 1336 \\
\text { miles. } \\
\text { - Reducir activos fijos por } \\
\text { US } \$ 375 \text { miles. }\end{array}$ & $\begin{array}{l}\text { - Acelerar cobranza y depuración de cuentas } \\
\text { vencidas (US } \$ 660 \text { miles). } \\
\text { - Administrar el aumento de cartera por } \\
\text { aumento de ventas (US \$ } 371 \text { miles). } \\
\text { - Acelerar reducción de inventarios, } \\
\text { optimizando tiempos y procesos de } \\
\text { producción. } \\
\text { - Simplificación de la gama de productos } \\
\text { producidos. } \\
\text { - Eliminar activos fijos obsoletos y en } \\
\text { desuso por US } \$ 375 \text { miles. }\end{array}$ \\
\hline Costo de Capital & $\begin{array}{l}\text { Reducir el costo de } \\
\text { capital }\end{array}$ & $\begin{array}{l}\text { - Reducir el costo de capital } \\
\text { de } 18 \text { a } 16.40 \% \text {. }\end{array}$ & $\begin{array}{l}\text { - Modificar la relación entre deuda y } \\
\text { patrimonio de } 1 \text { a } 1.5 \text { y pasar de } 1 \text { a } 1 \text {. } \\
\text { - Reducir el costo de deuda bancaria en } 1 \% \text {. }\end{array}$ \\
\hline
\end{tabular}


AMERICAN TEXTIL S.A.

El Árbol de Valor después de la Reestructuración

(expresado en miles de dólares)

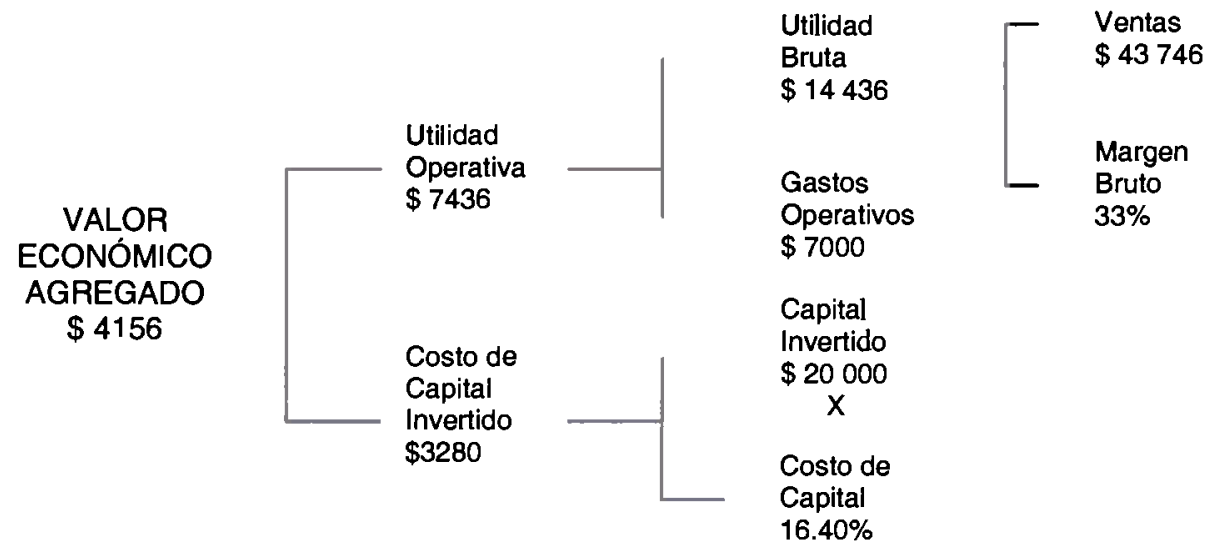

\section{CONCLUSIONES}

1. Una empresa orientada al éxito debe buscar aumentar su Valor Económico Agregado de manera sostenida en el tiempo.

2. La gestión empresarial y las finanzas tienen en el valor económico agregado un indicador financiero que vincula las estrategias con el objetivo financiero de maximizar la riqueza de los accionistas.

3. Para maximizar el Valor Económico Agregado, debe buscarse eficiencias operativa y financiera, a través de acciones estratégicas: aumentar las ventas, mejorar la rentabilidad bruta, reducir los gastos operativos, reducir el capital invertido y reducir el costo de capital.

4. Finalmente, para asegurar la implementación de estrategias financieras, la organización debe orientarse hacia una cultura de valor, implementando sistemas de información y control de gestión que miden el desempeño financiero y la política de incentivos a la administración hacia el logro del objetivo de crear valor para el accionista.

\section{REFERENCIAS}

AIMAR, Carlos. Estrategias y Realidades de Negocios. Ugerman (Editor), Argentina, 2000.

Bennett Stewart III. En busca del valor. Harper Collins. Publishers Inc., E.U.A., 1991.

CUEVAS VILLEGAS, Carlos. «Medición de desempeño». En: Revista Estudios Gerenciales, Colombia, Universidad ICESI, 2001.

ESCUELA DE ADMINISTRACIÓN DE NEGOCIOS PARA GRADUADOS. Convención Empresarial «Creación de Valor y Gerencia». Perú, ESAN ediciones, 1998.

FERNÁNDEZ, Pablo. "Valoración de Empresas». En: Gestión 2000. España, 1999.

LARRAGUIBEL, Marcelo. «Creación de Valon. En: Revista Administracióny Economia. Universidad Católica de Chile, 1999.

J. GITMAN, Lawrence. Principios de Administración Financiera. México, Edit. Addison Wesley Longman, 2000.

ORIOL, Amat. Valor Económico Agregado. Grupo Editorial Norma, España, 2000. 
Pontificia Universidad Católica del Perú. Foro «La Gerencia en el Perú en el siglo XXI». Lima, Editorial Centrum, 2000. RAPPAPORT, Alfred. La Creación de Valor para el Accionista. España, Ediciones Deusto, 1998.

Revista Business. «Compañias Top». Artículo sobre las Compañias más importantes según la lista de Forbes y Fortune, Perú, 2004.
SHAKED, Israel, MICHEL, Allen y LEROY, Pierre. "Creating Value through EVA-Myth or Reality». En: Revista Strategy \& Business, EVA. 1997.

STERN, Stewart. «La perspectiva Contable del EVA». En: Revista Journal of Applied Corporate Finance. EUA. 1998.

The Group of Lisboa. The MIT Press, Massachussets. «Limits to Competition», EUA, 1995. 